International Journal of Pure and Applied Mathematics

Volume 104 No. 2 2015, 193-202

ISSN: 1311-8080 (printed version); ISSN: 1314-3395 (on-line version)

url: http://www.ijpam.eu

doi: http://dx.doi.org/10.12732/ijpam.v104i2.4

\title{
EQUITABLE DOMINATING CHROMATIC SETS IN GRAPHS
}

\author{
L. Muthusubramanian ${ }^{1}$, S.P. Subbiah ${ }^{2}$, V. Swaminathan ${ }^{3}$ \\ ${ }^{1}$ Department of Mathematics \\ Sethu Institute of Technology \\ Kariapatti, Tamilnadu, INDIA \\ ${ }^{2}$ Department of Mathematics \\ Mannar Thirumalai Naicker College \\ Madurai, Tamilnadu, INDIA \\ ${ }^{3}$ Ramanujan Research Center in Mathematics \\ Saraswathi Narayanan College \\ Madurai, Tamilnadu, INDIA
}

\begin{abstract}
Let $G=(V, E)$ be a simple graph. A subset $D$ of $V(G)$ is said to be an equitable dominating set of $G$ if for every vertex $v \in V-D$ there exists a vertex $u \in D$ such that $u v \in E(G)$ and $|d(u)-d(v)| \leq 1$. A subset $D$ of $V(G)$ is said to be an equitable dominating chromatic set of $G$ if $D$ is an equitable dominating set of $G$ and $\chi(<D>)=\chi(G)$. Since $V$ is an equitable dominating chromatic set of $G$, the existence of equitable dominating chromatic set in a graph is guaranteed. The minimum cardinality of such a set is called the equitable dominating chromatic number of $G$ and is denoted by $\gamma_{c h}^{e}(G)$. The property of equitable dominating chromatic set is super hereditary. Hence equitable dominating chromatic set is minimal if and only if it is 1minimal. Characterization of minimal equitable dominating chromatic sets is derived. The values of $\gamma_{c h}^{e}(G)$ for many classes of graphs have been found. It is established that $1 \leq \gamma_{c h}^{e}(G) \leq n$. Interesting results are proved with respect to the new parameters.
\end{abstract}

Received: April 29, 2015

(C) 2015 Academic Publications, Ltd.

${ }^{\S}$ Correspondence author url: www.acadpubl.eu 
AMS Subject Classification: 05C17, 05C69, 05C70

Key Words: equitable dominating set, equitable dominating chromatic set

\section{Introduction}

The concept of Chromatic preserving sets was extensively studied by [3]. Further Chromatic preserving sets with specific property were considered. For example, Chromatic preserving strong(weak) dominating sets were defined and studied in [1]. Chromatic preserving transversals were introduced and studied by [5]. Degree Equitability is a concept introduced by Prof. E. Sampathkumar. Equitable dominating set was defined in [2]. In this paper, Equitable dominating chromatic sets are defined and studied.

Definition 1.1. A vertex and an edge are said to cover each other if they are incident. A set of vertices which covers all the edges of a graph is called a vertex cover of $G$. The smallest number of vertices in any vertex cover of $G$ is called the vertex covering number of $G$ and is denoted by $\alpha_{o}(G)$.

Definition 1.2. Let $G=(V, E)$ be a simple graph. A subset $D$ of $V(G)$ is said to be an equitable dominating set of $G$ if for every vertex $v \in V-D$ there exists a vertex $u \in D$ such that $u v \in E(G)$ and $|d(u)-d(v)| \leq 1$.

Definition 1.3. Let $G=(V, E)$ be a simple graph. A subset $D$ of $V(G)$ is said to be a dom-chromatic set or (dc-set) if $D$ is a dominating set of $G$ and $\chi(<D>)=\chi(G)$. The minimum cardinality of dom-chromatic set in a graph $G$ is called the dom-chromatic number or dc-number and is denoted by $\gamma_{c h}(G)$.

Definition 1.4. Let $G$ be a simple graph and let $D$ be a subset of $V(G)$. $D$ is said to be an equitable dominating chromatic set of $G$ if $D$ is an equitable dominating set of $G$ and $\chi(<D>)=\chi(G)$. The minimum cardinality of such a set is called the equitable dominating chromatic number of $G$ and is denoted by $\gamma_{c h}^{e}(G)$.

Since $V$ is an equitable dominating chromatic set of $G$, the existence of equitable dominating chromatic set in a graph is guaranteed.

\section{$\gamma_{\text {ch }}^{\text {e }}(\mathbf{G})$ for standard graphs}

1. $\gamma_{c h}^{e}\left(K_{n}\right)=n$ 
2. $\gamma_{c h}^{e}\left(\overline{K_{n}}\right)=n$

3. $\gamma_{c h}^{e}\left(K_{1, n}\right)=n+1, n \geq 1$

4. $\gamma_{c h}^{e}\left(P_{n}\right)=\gamma_{c h}\left(P_{n}\right)= \begin{cases}(n+3) / 3 & \text { if } n \equiv 0(\bmod 3), \\ (n+2) / 3 & \text { if } n \equiv 1(\bmod 3), \\ (n+1) / 3 & \text { if } n \equiv 2(\bmod 3),\end{cases}$

5. $\gamma_{c h}^{e}\left(C_{n}\right)=\gamma_{c h}\left(C_{n}\right)= \begin{cases}(n+3) / 3 & \text { if } n \equiv 0(\bmod 3), \\ (n+2) / 3 & \text { if } n \equiv 1(\bmod 3), \\ (n+1) / 3 & \text { if } n \equiv 2(\bmod 3),\end{cases}$

6. $\gamma_{c h}^{e}\left(W_{n}\right)= \begin{cases}3 & \text { if } n \text { is odd } \\ n & \text { if } n \text { is even }\end{cases}$

7. $\gamma_{c h}^{e}\left(K_{m, n}\right)= \begin{cases}2 & \text { if }|m-n| \leq 1, \\ m+n & \text { if }|m-n| \geq 2,\end{cases}$

8. $\gamma_{c h}^{e}\left(D_{r, s}\right)= \begin{cases}r+s+1 & \text { if }|r-s| \leq 1, r \text { (or) } s \geq 2, \\ r+s+2 & \text { if }|r-s| \geq 2 \\ r+s & \text { if } r=s=1\end{cases}$

9. $\gamma_{c h}^{e}\left(F_{n}\right)= \begin{cases}\left\lceil\frac{n}{3}\right\rceil+2 & \text { if } n \neq 1,4 \\ 2 & \text { if } n=1 \\ 3 & \text { if } n=4\end{cases}$

10. $\gamma_{c h}^{e}(P)=5=\gamma_{c h}(P)$.

Remark 1.5. Since any equitable dominating set is a dominating set, $\gamma_{c h}(G) \leq \gamma_{c h}^{e}(G)$.

Theorem 1.6. Let $D$ be an equitable dominating chromatic set of $G$. Every vertex in $V-D$ is not adjacent to at least one vertex of $D$.

Proof. Let $u \in V-D$. Suppose $u$ is adjacent with every vertex of $D$. Then $\chi(<D \cup\{u\}>)=\chi(<D>)+1$. Since $D$ is an equitable dominating chromatic set of $G, \chi(<D>)=\chi(G)$. Therefore, $\chi(<D \cup\{u\}>)=\chi(<G>)+1$. Since for any subgraph $S$ of $G, \chi(S) \leq \chi(G)$, we get a contradiction. Therefore, $u$ is not adjacent with some vertex of $D$. 
Remark 1.7. Let $G$ be a simple graph. Then $1 \leq \gamma_{c h}^{e}(G) \leq n$.

Remark 1.8. $\gamma_{c h}^{e}(G)=1$ if and only if $G=K_{1}$.

Theorem 1.9. Let $G$ be an equitable graph without isolates. (That is, Given $u, v$ in $V(G)$ with $u v \in E(G)$ then $|\operatorname{deg}(u)-\operatorname{deg}(v)| \leq 1)$. Then $\gamma_{c h}^{e}(G)=n$ if and only if $G$ is a $\chi$-critical graph.

Proof. Suppose $G$ satisfies the hypothesis. Suppose $G$ is a $\chi$-critical graph. Then for any equitable dominating chromatic set $D$ of $G, \chi(<D>)=\chi(G)$. Since $G$ is $\chi$-critical graph, $D=V(G)$. Therefore $\gamma_{c h}^{e}(G)=|D|=n$. Conversely, Suppose $\gamma_{c h}^{e}(G)=n$. Let $D$ be a minimum equitable dominating chromatic set of $G$. Then $|D|=n$. If $G$ is totally disconnected then $\gamma_{c h}^{e}(G)=n$ and $G$ is $\chi$-critical. Suppose $G$ is not totally disconnected. Then there exist vertices $u, v$ which are adjacent. Since $G$ is equitable $|d(u)-d(v)| \leq 1$. Therefore, $G-\{u\}$ is an equitable dominating set of $G$. Since $\gamma_{c h}^{e}(G)=n$, $G-\{u\}$ is not a dominating chromatic set. Therefore, $\chi(<G-\{u\}>)<\chi(G)$. Let $H$ be a proper subgraph of $G$. Let $u \notin V(H)$. Then $H \subseteq G-\{u\}$ and $\chi(H) \leq \chi(G-\{u\})<\chi(G)$. Suppose $u \in V(H)$. Let $w \in H$. If $w$ is not adjacent with any vertex in $H$, then $w$ is adjacent with some vertex $x$ in $G$, $x \notin H . H \subseteq G-\{x\}$. Since $G-\{x\}$ is equitable dominating set of $G$, $\chi(G-\{x\})<\chi(G)$. Therefore, $\chi(H)<\chi(G)$. Suppose $N(H) \subseteq H$. Then $H$ is not a dominating set of $G$, since $H$ is a proper subgraph of $G$ and $N(H) \subseteq H$. Let $y \in V-V(H)$. Then $y$ is adjacent with some $z \in V-V(H)$. Therefore, $G-\{y\}$ is an equitable dominating set of $G$. If $G-\{y\}$ is an equitable dominating chromatic set of $G$, then $\gamma_{c h}^{e}(G) \leq \gamma_{c h}^{e}(G-\{y\})$. Since $\gamma_{c h}^{e}(G)=n$, $\gamma_{c h}^{e}(G-\{y\})=n$. But $|V(G-\{y\})| \leq n-1$, a contradiction. Therefore, $\chi(H) \leq \chi(G-\{y\})<\chi(G)$. Therefore, $G$ is a $\chi$-critical graph.

Remark 1.10. Suppose $G$ is a disconnected graph with $\gamma_{c h}^{e}(G)=n$. Then either $G$ is $\overline{K_{n}}$ or $G$ has exactly one non trivial component which is $\chi$-critical, equitable and without isolates.

Let $G$ be a disconnected graph with $\gamma_{c h}^{e}(G)=n$. If every component of $G$ is trivial, then $G=\overline{K_{n}}$. Suppose there exist two or more components of $G$ which are non trivial. Let $G_{1}$ and $G_{2}$ be two components of $G$ which are non trivial. Let $\chi\left(G_{1}\right) \geq \chi\left(G_{2}\right)$. Let $D_{1}$ be a $\gamma_{c h}^{e}$-set of $G_{1}$ and $D_{2}$ be a $\gamma^{e}$-set of $G_{2}$. Then $D_{1} \cup D_{2}$ is a $\gamma_{c h}^{e}$-set of $G_{1} \cup G_{2}$. For: $D_{1} \cup D_{2}$ is an equitable dominating set 
of $G_{1} \cup G_{2}$ of minimum cardinality,

$$
\begin{aligned}
\chi\left(D_{1} \cup D_{2}\right) & =\max \left\{\chi\left(D_{1}\right), \chi\left(D_{2}\right)\right\} \\
& =\max \left\{\chi\left(G_{1}\right), \chi\left(G_{2}\right)\right\} \\
& =\chi\left(G_{1}\right) \quad\left(\text { Since } \chi\left(D_{2}\right) \leq \chi\left(G_{2}\right) \leq \chi\left(G_{1}\right)\right) \\
& =\chi\left(G_{1} \cup G_{2}\right) .
\end{aligned}
$$

Therefore, $D_{1} \cup D_{2}$ is a $\gamma_{c h}^{e}$-set of $G_{1} \cup G_{2}$. $\left|D_{2}\right| \leq\left|V\left(G_{2}\right)\right|-1$ (since $G_{2}$ has no equitable isolates). Therefore, $\gamma_{c h}^{e}\left(G_{1} \cup G_{2}\right)<\left|V\left(G_{1}\right)\right|+\left|V\left(G_{2}\right)\right|$, a contradiction. (since $\gamma_{c h}^{e}(G)=n$ ). Therefore, there exists exactly one component of $G$ which is non trivial. Let $G_{1}$ be a non trivial component of $G$. Therefore, $G=G_{1} \cup t K_{1}$ where $G_{1}$ is a non trivial component. Therefore, $n=\gamma_{c h}^{e}(G)=$ $\gamma_{c h}^{e}\left(G_{1} \cup t K_{1}\right)=t+\gamma_{c h}^{e}\left(G_{1}\right)$. Therefore, $\gamma_{c h}^{e}\left(G_{1}\right)=n-t=\left|V\left(G_{1}\right)\right|$. Therefore, $G_{1}$ is $\chi$-critical. Therefore, If $\gamma_{c h}^{e}(G)=n$, then $G$ is either $\overline{K_{n}}$ or $G$ has exactly one non trivial component which is $\chi$-critical and which is equitable without isolates.

Remark 1.11. The converse of the above remark is also true.

For: If $G=\overline{K_{n}}, \gamma_{c h}^{e}(G)=n$. If $G$ has exactly one non trivial component say $G_{1}$ which is $\chi$-critical,equitable and without isolates. Then $\gamma_{c h}^{e}\left(G_{1}\right)=\left|V\left(G_{1}\right)\right|$. Therefore, $\gamma_{c h}^{e}(G)=\left|V\left(G_{1}\right)\right|+t$ where $t$ is the number of trivial components of G. Therefore, $\gamma_{c h}^{e}(G)=|V(G)|=n$.

Remark 1.12. Any superset of an equitable dominating chromatic set of $G$ is also an equitable dominating chromatic set of $G$. That is, equitable dominating chromatic property is super hereditary. Therefore, an equitable dominating chromatic set is minimal if and only if it is 1-minimal.

Theorem 1.13. Let $D$ be an equitable dominating chromatic set of $G$. $D$ is minimal if and only if for each $u \in D$, one of the following holds:

(i) $N(u) \cap D=\phi$ (or) $|d(u)-d(v)| \geq 2$ for all $v \in N(u) \cap D$;

(ii)There exists a vertex $v \in V-D$ such that $N(v) \cap D=\{u\}$ and $\mid d(u)-$ $d(v) \mid \leq 1$

(iii) $\chi(D-\{u\})<\chi(G)$.

Proof. Suppose $D$ is a minimal equitable dominating chromatic set of $G$. Since the property of equitable chromatic domination is super hereditary, $D$ is minimal if and only if $D-\{u\}$ is not an equitable dominating chromatic set for 
any $u \in D$. Therefore, either $\chi(D-\{u\})<\chi(G)$ or $D-\{u\}$ is not an equitable dominating set (ie) $N(u) \cap D=\phi$ or $|d(u)-d(v)| \geq 2$ for all $v \in N(u) \cap D$ or there exists a vertex $v \in V-D$ such that $N(v) \cap D=\{u\}$ and $|d(u)-d(v)| \leq 1$. Conversely, Suppose $D$ is an equitable dominating chromatic set such that for every $u \in D$ one of the above three conditions is satisfied. Consider $D-\{u\}$. If $u$ satisfies $(i)$ then either $u$ is not dominated by any vertex of $D-\{u\}$ or $u$ is not equitably dominated by any vertex of $D-\{u\}$. If $u$ satisfies $(i i)$ then $u$ has an equitable private neighbor in $V-D$ and hence $D-\{u\}$ is not an equitable dominating set. If $u$ satisfies $(i i i)$ then $D-\{u\}$ does not preserve the chromaticity of $G$. Hence $D-\{u\}$ is not an equitable dominating chromatic set if satisfies any one of the three conditions. Hence the theorem.

Theorem 1.14. Let $D$ be an equitable dominating chromatic set of $G$. Then

$$
|V-D|=\sum_{u \in D} \operatorname{deg}(u)
$$

if and only if $G=\overline{K_{n}}$.

Proof. If $G=\overline{K_{n}}$ then $D=V$ and $\operatorname{deg}(u)=0$ for every $u \in D$. Therefore, $|V-D|=|V-V|=0=\sum_{u \in D} \operatorname{deg}(u)$. Suppose $G \neq \overline{K_{n}}$. Then $G$ has an edge and hence $\chi(G) \geq 2$. Therefore, $\chi(<D>) \geq 2$. Therefore, $\langle D\rangle$ has an edge. Therefore, $\sum_{u \in D} \operatorname{deg}(u) \geq 2$. Since $D$ is an equitable dominating set, each vertex in $V-D$ is adjacent to at least one vertex in $D$. Therefore, $\sum_{u \in D} \operatorname{deg}(u) \geq|V-D|+2$. Hence $|V-D| \neq \sum_{u \in D} \operatorname{deg}(u)$. Hence the theorem.

Corollary 1.15. For any non trivial connected graph, $\sum_{u \in D} \operatorname{deg}(u) \geq \mid V-$ $D \mid+2$ where $D$ is a dominating chromatic set of $G$.

Theorem 1.16. For any graph $G,\left\lfloor\frac{n}{\Delta(G)+1}\right\rfloor \leq \gamma_{c h}^{e}(G)$ and equality holds if and only if $G=\overline{K_{n}}$.

Proof. Since $\left\lfloor\frac{n}{\Delta(G)+1}\right\rfloor \leq \gamma(G) \leq \gamma_{c h}^{e}(G)$, the lower bound is attained. If $G=\overline{K_{n}}$ then $\gamma_{c h}^{e}(G)=n .\left\lfloor\frac{n}{\Delta(G)+1}\right\rfloor=\left\lfloor\frac{n}{0+1}\right\rfloor=n$. Therefore, $\gamma_{c h}^{e}(G)=$ $\left\lfloor\frac{n}{\Delta(G)+1}\right\rfloor$. Suppose $\left\lfloor\frac{n}{\Delta(G)+1}\right\rfloor=\gamma_{c h}^{e}(G)=k($ say $)$. Suppose $G \neq \overline{K_{n}}$. Then from 
the above corollary, $|V-D|<\sum_{u \in D} \operatorname{deg}(u)$ (i.e) $n-k<\sum_{u \in D} \operatorname{deg}(u) \leq k \Delta(G)$. Therefore, $n<k(\Delta(G)+1)$.

$$
\begin{gathered}
\frac{n}{\Delta(G)+1}<k \\
\left\lfloor\frac{n}{\Delta(G)+1}\right\rfloor<k
\end{gathered}
$$

Therefore, If $G \neq \overline{K_{n}}$ then $\left\lfloor\frac{n}{\Delta(G)+1}\right\rfloor<\gamma_{c h}^{e}(G)$. Hence the theorem.

Remark 1.17. The above result is true even when equitability is dropped.

Theorem 1.18. Given a positive integer $k$ there exists a graph $G$ such that $\gamma_{c h}^{e}(G)=k$.

Proof. Suppose $k=1$. Then $\gamma_{c h}^{e}\left(K_{1}\right)=1=k$. Let $k \geq 2$. Let $G=K_{k}$. Then $\gamma_{c h}^{e}\left(K_{k}\right)=k$. Also, $\gamma_{c h}^{e}\left(K_{k-1,1}\right)=k, k \geq 3$.

Theorem 1.19. Given a positive integer $k$ there exists a graph $G$ such that:

(i) $\gamma_{c h}^{e}(G)-\gamma(G)=k$;

(ii) $\gamma_{c h}^{e}(G)-\gamma^{e}(G)=k$;

(iii) $\gamma_{c h}^{e}(G)-\gamma_{c h}(G)=k$.

Proof. (i) Let $G=K_{k+1}$. Then $\gamma_{c h}^{e}(G)=k+1$. But $\gamma(G)=1$. Therefore, $\gamma_{c h}^{e}(G)-\gamma(G)=k$.

(ii) Let $G=K_{k+1}^{+} \cdot \gamma^{e}(G)=k+1+1=k+2 . \gamma_{c h}^{e}(G)=2(k+1)=2 k+2$. Therefore, $\gamma_{c h}^{e}(G)-\gamma^{e}(G)=2 k+2-(k+2)=k$.

(iii) Let $G=K_{k}^{+} \cdot \gamma_{c h}^{e}(G)=2 k, \gamma_{c h}(G)=k$.

Therefore, $\gamma_{c h}^{e}(G)-\gamma_{c h}(G)=2 k-k=k$.

Theorem 1.20. If $G$ is triangle free with $\chi(G) \geq 3$ then $\gamma_{c h}(G) \geq 5$ and hence $\gamma_{c h}^{e}(G) \geq 5$.

Proof. Let $D$ be a $\gamma_{c h}$ set of $G$. Then $\chi(<D>)=\chi(G) \geq 3$. Since $G$ is triangle free,$\langle D\rangle$ is also triangle free. If $\langle D\rangle$ contains no odd cycle 
then $\langle D\rangle$ is bipartite and hence $\chi(<D>)=2$, a contradiction. Therefore, $<D>$ contains an odd cycle of length greater than or equal to 5 . Therefore, $|D| \geq 5$. $\gamma_{c h}(G) \geq 5$. Therefore, $\gamma_{c h}^{e}(G) \geq \gamma_{c h}(G) \geq 5$.

Theorem 1.21. Let $G$ be an equitable graph without isolates and Let $G$ be $\chi$-critical. Then $\alpha_{0}(G)<\gamma_{c h}^{e}(G)$.

Proof. For any graph $G, \alpha_{0}(G) \leq n-1$. If $G$ is $\chi$-critical and equitable without isolates, $\gamma_{c h}^{e}(G)=n$. Therefore, $\alpha_{0}(G)<\gamma_{c h}^{e}(G)$.

Theorem 1.22. If $G$ is $\chi$-critical and equitable without isolates, and $\operatorname{diam}(G) \geq 2$. Then $\alpha_{0}(G)+2 \leq \gamma_{c h}^{e}(G)$.

Proof. Since $\operatorname{diam}(G) \geq 2, \beta_{0}(G) \geq 2 . \alpha_{0}(G) \leq n-2$. Therefore, $\alpha_{0}(G)+$ $2 \leq n=\gamma_{c h}^{e}(G)$.

Theorem 1.23. Let $G$ be a perfect graph. Then $\gamma_{c h}^{e}(G) \leq \gamma^{e}(G)+\omega(G)$.

Proof. Let $S$ be a maximum clique in $G$ and $D$ a $\gamma^{e}$-set of $G$. Since $G$ is perfect, $\omega(G)=\chi(G)$. Therefore, $\chi(G)=|S|=\chi(<S\rangle)=\chi(\langle S \cup D\rangle)$. Since equitable domination is super hereditary, $S \cup D$ is an equitable dominating set of $G$. Since $\chi(\langle S \cup D>)=\chi(G),\langle S \cup D>$ is an equitable dominating chromatic set of $G$. Therefore, $\gamma_{c h}^{e}(G) \leq|S \cup D| \leq|S|+|D|=\omega(G)+\gamma^{e}(G)$.

Remark 1.24. There exists a graph $G$, such that $\gamma_{c h}^{e}(G)=\gamma^{e}(G)+\omega(G)$. For: Consider the Petersen graph $P . \gamma_{c h}^{e}(P)=5, \gamma^{e}(P)=3, \omega(P)=2$.

Therefore, $\gamma_{c h}^{e}(P)=\gamma^{e}(P)+\omega(P)$.

Remark 1.25. Let $D$ be a graph with a full degree vertex. Then $\gamma_{c h}^{e}(G)$ need not be equal to $\chi(G)$.

For example, $\gamma_{c h}^{e}\left(D_{r, s}\right)=r+s+1$ where $|r-s| \leq 1, r($ or $) s \geq 2$.

Therefore, $\gamma_{c h}^{e}\left(D_{r, s}\right) \neq \chi\left(D_{r, s}\right)=2$.

Theorem 1.26. Let $G$ be a perfect graph with a full degree vertex which equitably dominates all other vertices. Then $\gamma_{c h}^{e}(G)=\chi(G)$.

Proof. Let $u$ be a full degree vertex of $G$ such that $u$ equitably dominates every other vertex of $G$. Let $S$ be a maximum clique in $G$. Then $u \in S$. 
$|S|=\omega(G)=\chi(G)$. Since $S$ is an equitable dominating chromatic set of $G$, $\gamma_{c h}^{e}(G) \leq|S|=\chi(G)$. But $\chi(G) \leq \gamma_{c h}^{e}(G)$. Therefore, $\gamma_{c h}^{e}(G)=\chi(G)$.

Example 1.27. $K_{1, n}$ is a perfect graph with a full degree vertex. If $n \geq 3$, the full degree vertex does not equitably dominate other vertices. Also, $\gamma_{c h}^{e}(G)=n+1$ and $\chi(G)=2$. Therefore, $\gamma_{c h}^{e}(G)>\chi(G)$.

Example 1.28. $K_{1,2}$ is a perfect graph with a full degree vertex which equally dominates the other two vertices. Here, $\chi\left(K_{1,2}\right)=2, \gamma_{c h}^{e}\left(K_{1,2}\right)=2$.

Theorem 1.29. There exists a connected graph $G$ and a graph $G^{1}$ such that $G^{1}$ is obtained from $G$ by adding exactly one vertex and $\gamma_{c h}^{e}\left(G^{1}\right)-\gamma_{c h}^{e}(G)=$ 1.

Proof. Let $G=P_{3(N+2)}$. Then $\gamma_{c h}^{e}(G)=(3(N+2)+3) / 3=N+3$. Let $G^{1}$ be the graph obtained from $G$ by adding a new vertex $v$ and joining it to all the vertices of $G$. $\gamma_{c h}^{e}\left(G^{1}\right)=\gamma_{c h}^{e}(G)+1$. Hence $\gamma_{c h}^{e}\left(G^{1}\right)-\gamma_{c h}^{e}(G)=1$.

Corollary 1.30. Given a positive integer $N$, there exists a graph $G$ and a graph $G^{!}$obtained from $G$ by adding exactly $N$ vertices such that $\gamma_{c h}^{e}\left(G^{1}\right)-$ $\gamma_{c h}^{e}(G)=N$.

Proof. Take a path of $3(N+2)$ vertices and add $N$ vertices and make them adjacent with every vertex of the path. Then the result follows.

Theorem 1.31. Let $G$ be a bipartite graph and let $G \neq \overline{K_{n}}$. Then $\gamma^{e}(G) \leq \gamma_{c h}^{e}(G) \leq \gamma^{e}(G)+1$.

Proof. Since $G$ is bipartite and $G \neq \overline{K_{n}}, \chi(G)=2$. Let $D$ be a minimum equitable dominating set of $G$. Then $|D|=\gamma^{e}(G)$. If $\langle D>$ contains an edge then $\chi(\langle D\rangle)=2$ and hence $\langle D\rangle$ is an equitable dominating chromatic set of $G$. Therefore, $\gamma_{c h}^{e}(G)=\gamma^{e}(G)$. Suppose $\langle D\rangle$ is totally disconnected for every minimum equitable dominating sets of $G$, then $D \cup\{v\}$ is a $\gamma_{c h}^{e}$-set of $G$ where $v \in V-D$. Therefore, $\gamma_{c h}^{e}(G)=|D|+1=\gamma^{e}(G)+1$.

\section{References}

[1] S. Balamurugan, A. Wilson Baskar, V. Swaminathan, Equality of strong 
domination and chromatic domination in graphs, International Journal of Mathematics and Soft Computing, 1, No. 1 (2011), 69-76.

[2] K.M. Dharmalingam, V. Swaminathan, Degree equitable domination in graphs, Kragujevac Journal of Mathematics, 35, No. 1 (2011), 191-197.

[3] T.N. Janakiraman, M. Poopalaranjani, On Some Coloring and Domination Parameters in Graphs, Ph.D Thesis, Bharathidasan University, Trichy (2006).

[4] P. Jeyaprakash, V. Swaminathan, $\tau_{c}$-Sets in a tree, International Journal of Pure And Applied Mathematics, 91, No. 1 (2014), 43-47.

[5] P. Jeyaprakash, V. Swaminathan, Clique transversal sets, International Journal of Mathematics and Soft Computing, 3, No. 2 (2013), 21-25. 